\title{
PMU-based Line Differential Protection under GPS Spoofing Attack
}

\author{
Jiahao Xie \\ Georgia Institute of Technology \\ orbitx@gatech.edu
}

\author{
A. P. Sakis Meliopoulos \\ Georgia Institute of Technology \\ sakis.m@gatech.edu
}

\author{
George Cokkinides \\ Georgia Institute of Technology \\ cokkinides@comcast.net
}

\begin{abstract}
Many power grid applications rely on the timesynchronized measurement data collected by phasor measurement units (PMUs) and or Merging Units $(M U s))$. The synchronization of data from multiple $P M U$ s and MUs relies on the global positioning system (GPS) to provide the time reference. The civilian GPS receivers used in PMU operate according to the publicly available mechanism. Therefore, attackers can easily generate false GPS signals with low-cost portable devices. During the spoofing attacks, the signal received by the PMUs and MUs is arbitrarily modified by the attacker without being detected, leading to the malfunction of other applications in the power system including transmission line protection. In this paper, we investigate the effect of GPS spoofing attack on transmission line differential protection schemes based on PMUs, including the percentage differential method and alpha-plane protection method. The mis-operation of these relays is observed during GPS spoofing attacks. In addition, we propose a quasi-dynamic stateestimation-based method to detect GPS spoofing attacks, identify the affected PMUs, correct the compromised data, and enable the continuous and reliable operation of the relays. Numerical results show that the proposed method detects the GPS spoofing successfully and the recovered measurement data eliminate the misoperation of line differential protection.
\end{abstract}

\section{Introduction}

The Global Positioning System (GPS), is designed to provide accurate location and time for various civil and military applications around the world. GPS receivers calculate the local coordination and time based on the signal transmitted from multiple satellites. For instance, the Phasor Measurement Units (PMUs) network, which is widely used in power grids [1], relies on the GPS to produce time-synchronized measurement. Most of the PMUs utilize the civilian version of the GPS signal[2], which is much less encrypted than the military GPS signal. The weakness in the encryption can be utilized by malicious attackers to fabricate false signals.
For example, attackers can change the time reference by an attacker-selected offset. The target will receive the contaminated signal with a wrong time reference. The attacker starts with transmitting a replica of the genuine GPS signal. Next, the spoofing signal gradually drifts from the genuine signal. Eventually, the attacker can control the target without being detected. Reference [3] presents a portable civilian GPS spoofer using low-cost hardware such as digital signal processors and a software-based spoofing module. In addition, the spoofer is small enough to be placed near the antenna of the victim. Reference[4] presents the outcome of GPS spoofing attack generating resonant oscillations in the HVDC system. The possible outcome of GPS spoofing attacks on PMUs includes the PMU clock offset attackresearchers [5] formulate an optimization scheme to achieve an attack to manipulate the PMU clocks while avoiding detection by abrupt or inconsistent changes in the signals, regardless of the number of visible satellites.

PMU applications in power grids include multiple aspects[6]-[8]: power system monitoring such as state estimation; power system control, such as damping electromechanical oscillations; The hardware-in-theloop experiment in reference [9] proves that the power control functions can be compromised during a GPS spoofing attack. Another application of PMU is power system protection, such as transmission line protection [10]. One of the line protection schemes is line differential protection. Differential protection relies heavily on the synchronization of measurement data from different locations. There are two methods[11] to conduct the synchronization: channel-based and external time reference based. The former uses the knowledge of time delay in the channel, it is an approximate method and performs poorly when the channel is not symmetrical. The latter usually use GPS in practice. For instance, a pair of PMUs can be placed at the terminals of the transmission line, and the sum of current phasor measurements are used to detect the possible fault inside the line. For a line with low line charging, the sum is always close to zero under the normal operating state. The GPS spoofing attack, however, will alter the phase angle of the current phasor and will affect the sum. The line may be tripped by the 
relay even though there is no fault in the line. Therefore, the attackers can initiate a misoperation via GPS spoofing techniques. Precision Time Protocol (PTP) is able to synchronize the clocks in multiple devices inside the substations[12]. The use of PTP does not alleviate the effects of a GPS spoofing attack. The use of PTP to synchronize different substations long distances apart is not practical and cost-prohibitive. Another possible outcome of the GPS spoofing attack is the shift of frequency measurements in PMUs. Researchers[13] propose to detect GPS spoofing via calculations based on PMU frequency measurements.

In this paper, we investigate the performance of the line differential protection scheme under GPS spoofing attacks, including the percentage differential method and alpha-plane protection method. GPS spoofing attacks with different attack angles are simulated to study the possibility of misoperation. We also propose a three-phase quasi-dynamic state estimation based method[14], [15] to detect GPS spoofing attacks and recover the actual measurement data from the compromised data. This state-estimation-based method work with electrical and mechanical measurements and physically based device models to monitor the state of the power system. It has the additional capability of identifying the compromised PMUs as well as the source of the altered timing signals and therefore it is useful in containing and repairing the attack. This method is proved to be able to detect spoofing attacks with high sensitivity. In addition, the estimated state and the system model are utilized to recover the actual measurement during the spoofing attack. This paper aims to illustrate that with the recovered measurements data from state estimation, the misoperation of line differential protection scheme can be avoided and ensure the reliability of the relays. The rest of the paper is organized as follows. Section 2 describes how GPS spoofing attacks the output of PMUs. Section 3 introduces the performance of percentage line differential protection during GPS spoofing attacks. Section 4 describes the effect of the spoofing attack on alpha-plane line differential protection. Section 5 presents the quasi-dynamic state-estimation-based algorithm used in the attack detection and mitigation method. A numerical example demonstrates that the proposed method eliminates relay misoperations. Section 6 summarizes the performance of the relays and effectiveness of the mitigation method.

\section{PMU under GPS Spoofing Attack}

Phasor measurement units collect and stream measured phasor values with timestamps. Inside PMUs, GPS signals are used as the time reference to synchronously sample waveforms and assign the time stamp to the output phasors (best performing PMUs have a typical accuracy of $1 \mu \mathrm{s})$. As mentioned in the previous section, the existence of GPS spoofing attacks severely reduces the reliability of the GPS signal [16]. As a result, the GPS signal received by PMU could be contaminated and subject to any modification from the attacker. The attack studied in this paper is to delay the authentic GPS signal by a specific time delay $t_{\text {delay }}$, which is depicted in Figure 1. A sophisticated GPS spoofing attack transmits fabricated GPS signals, which may appear genuine but will shift the time reference in the receiver without being detected. In this case, the time shift in the clock of PMU will translate into a phase shift in the output phasor. All voltage measurements and current measurements that use the spoofed GPS receiver will exhibit a delay angle $\theta_{\text {delay }}$. An example of this process is shown in Equation (1):

$\tilde{I}_{21}^{m}(t)=\tilde{I}_{21}(t) \cdot \exp \left(j t_{\text {delay }} \omega_{0}\right)=\tilde{I}_{21}(t) \cdot \exp \left(j \theta_{\text {delay }}\right)$

where $\omega_{0}$ is the angular frequency of the system, $\tilde{I}_{21}(t)$ are the phasors that would have been computed without the assumed GPS spoofing and $\tilde{I}_{21}^{m}(t)$ are the phasors computed with the GPS spoofed signal.

Two line differential protection schemes are discussed and investigated in this paper: percentage differential and alpha plane differential.

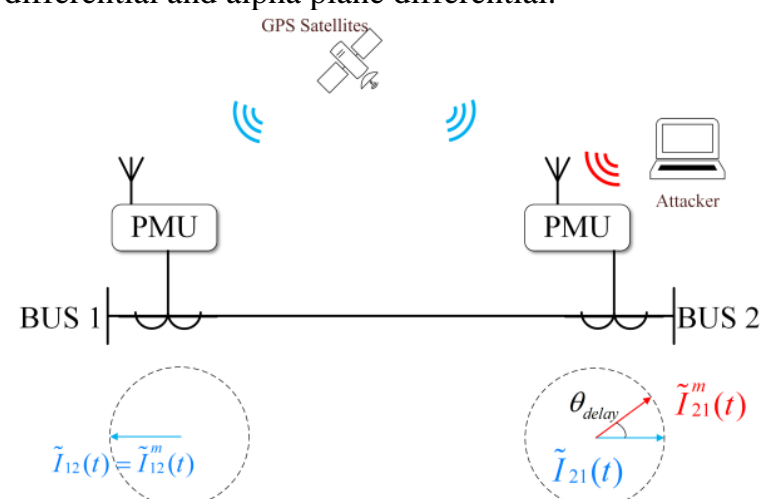

Figure 1: GPS Spoofing Attack Illustration

\section{Percentage Line Differential Protection}

The introduction of GPS synchronized measurements and fiber optic communications greatly reduce the complexity of differential protection of transmission lines. Specifically, GPS synchronized measurements enable the simultaneous measurement of voltages and currents at remote terminals of a line. These measurements are time-tagged and are communicated via fiberoptic to the location of the relay. The relay can time align the measurements and perform a differential protection function with minimal interference from the communication latency. However, the GPS spoofing attack will largely reduce the accuracy of the time stamp and the misoperation of relays is possible. In this section, the percentage line differential 
protection method is investigated during the GPS spoofing attack.

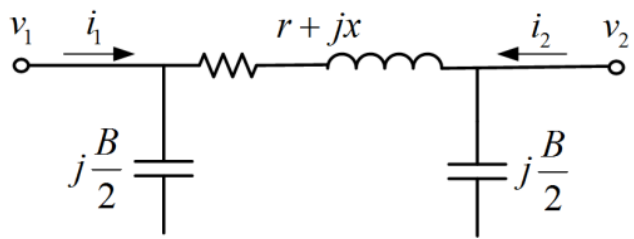

Figure 2: Simplified Line Model

The operating current $I_{o p}$ and restraining current $I_{r e s}$ are two key metrics in the percentage differential protection, they are calculated as:

$$
\begin{gathered}
I_{o p}=\left|i_{1}+i_{2}\right| \\
I_{\text {res }}=\frac{1}{2}\left(\left|i_{1}\right|+\left|i_{2}\right|\right)
\end{gathered}
$$

The currents $i_{1}, i_{2}$ are the current flowing into the transmission line at the two terminals. To derive simplified expressions, a simplified transmission line model (pi-model) is used as shown in Figure 2. The impedance of the line equals $r+j x$ and the line charging is $j B$. Therefore, the current can be calculated as

$$
\begin{gathered}
y=1 /(r+j x) \\
i_{1}=y\left(v_{1}-v_{2}\right)+j \frac{B}{2} v_{1} \\
i_{2}=y\left(v_{2}-v_{1}\right)+j \frac{B}{2} v_{2} y=1 /(r+j x)
\end{gathered}
$$

The percentage differential relay will trip if:

$$
\begin{gathered}
I_{o p}>K_{1} I_{R} \\
\text { AND } I_{o p} / I_{r e s}>K_{2}
\end{gathered}
$$

where $I_{R}$ is the rated current of the transmission line. $K_{1}, K_{2}$ are user-defined constants.

Assume the target of the GPS spoofing attack is substation 2 and the attack angle is $\theta_{s}$, the current measurement at substation 2 becomes $i_{2}^{\prime}=i_{2} e^{j \theta_{s}}$. As a result, the operating current and restraining current are calculated as:

$$
\begin{aligned}
& I_{o p}^{\prime}=\left|i_{1}+i_{2}^{\prime}\right|=\left|y\left(v_{1}-v_{2}\right)\left(1-e^{j \theta_{s}}\right)+j \frac{B}{2} v_{1}+j \frac{B}{2} v_{2} e^{j \theta_{S}}\right| \\
& I_{r e s}^{\prime}=\frac{1}{2}\left(\left|i_{1}\right|+\left|i_{2}^{\prime}\right|\right) \\
& =\frac{1}{2}\left|y\left(v_{1}-v_{2}\right)+j \frac{B}{2} v_{1}\right|+\frac{1}{2}\left|\left(y\left(v_{2}-v_{1}\right)+j \frac{B}{2} v_{2}\right) e^{j \theta_{s}}\right| \\
& =\frac{1}{2}\left|y\left(v_{1}-v_{2}\right)+j \frac{B}{2} v_{1}\right|+\frac{1}{2}\left|y\left(v_{2}-v_{1}\right)+j \frac{B}{2} v_{2}\right|=I_{\text {res }}
\end{aligned}
$$

\subsection{Line charging is minimal}

First, the case with no line charging is studied. If $B \approx 0$ (short line), the magnitude of current in the line equals rated current, and there is no fault inside the line, then:

$$
\begin{gathered}
I_{o p}^{\prime}=\left|y\left(v_{1}-v_{2}\right)\left(1-e^{j \theta_{S}}\right)\right|=I_{R}\left|1-e^{j \theta_{S}}\right| \\
\frac{I_{o p}^{\prime}}{I_{r e s}^{\prime}}=\frac{\left|y\left(v_{1}-v_{2}\right)\left(1-e^{j \theta_{S}}\right)\right|}{\frac{1}{2}\left|y\left(v_{1}-v_{2}\right)\right|+\frac{1}{2}\left|y\left(v_{2}-v_{1}\right)\right|}=\left|1-e^{j \theta_{S}}\right|
\end{gathered}
$$

The percentage line differential will trip if expression (2) and expression (3) are satisfied.

Expression (2):

$$
\begin{gathered}
I_{o p}^{\prime}>K_{1} I_{R} \\
\left|1-e^{j \theta_{s}}\right|>K_{1} \\
\theta_{k 1}<\theta_{s}<2 \pi-\theta_{k 1}
\end{gathered}
$$

where $\theta_{k 1}=\operatorname{acos}\left(1-K_{1}^{2} / 2\right)$

Expression (3):

$$
\begin{gathered}
I_{o p}^{\prime} / I_{r e s}^{\prime}>K_{2} \\
\left|1-e^{j \theta_{s}}\right|>K_{2} \\
\theta_{k 2}<\theta_{s}<2 \pi-\theta_{k 2}
\end{gathered}
$$

where $\theta_{k 2}=\arccos \left(1-K_{2}^{2} / 2\right)$.

If $K_{1}=0.05, K_{2}=0.2$,

then $\theta_{k 1}=0.05 \mathrm{rad}, \theta_{k 2}=0.2 \mathrm{rad}$

In this case, if the attack angle is in the range $0.2<\theta_{s}<2 \pi-0.2$, then the percentage line differential relay will disconnect the line from the grid even though there is no fault. Therefore, the misoperation occurs due to the GPS spoofing attack.

\subsection{Line charging is not minimal}

If line charging is not minimal, the value of B will affect the response of the relay to the attack. In this section, the following parameters are used to calculate it numerically.

$$
\begin{gathered}
y=1 /(0.01+0.08 i) \\
v_{2}=1.0 \\
v_{1}=v_{2} e^{0.5 i} \\
I_{R}=\left|y\left(v_{1}-v_{2}\right)\right|
\end{gathered}
$$

The value of B changes from 0 to $0.4(\mathrm{pu})$. And the value of $I_{o p}^{\prime} / I_{R}$ and $I_{o p}^{\prime} / I_{\text {res }}^{\prime}$ in terms of $\theta_{s}$ are shown in Figure 3 and Figure 4 correspondingly. The region above the threshold (dotted line) corresponds to the attack angle that leads to the misoperation of the relay. 


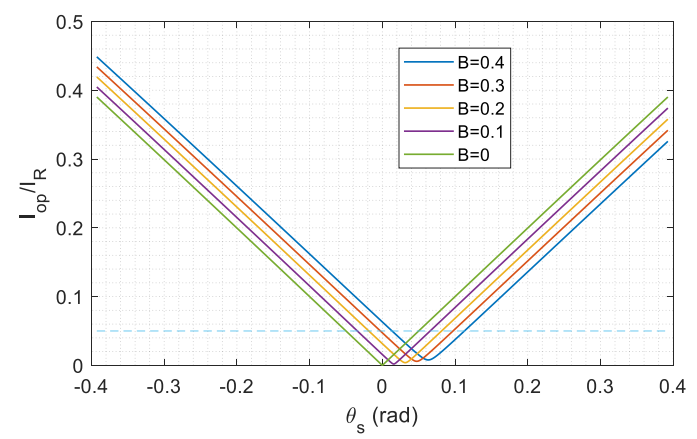

Figure 3: Percentage Line Differential $I_{o p}^{\prime} / I_{R}$

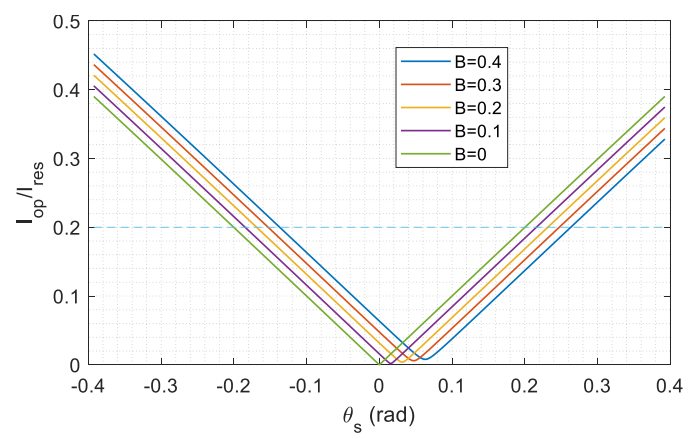

Figure 4: Percentage Line Differential $I_{o p}^{\prime} / I_{\text {res }}^{\prime}$

Similarly, we can present the results in terms of the length of the line $l(\mathrm{~km})$. The parameters used are:

$$
\begin{gathered}
y(l)=1 /((0.025+0.2 i) l) \\
B(l)=0.001 l \\
v_{2}(l)=1.0 \\
v_{1}(l)=v_{2}(l) \exp (0.001 i * l) \\
I_{R}(l)=\left|y(l)\left(v_{1}(l)-v_{2}(l)\right)\right|
\end{gathered}
$$

The length of the line changes from $10 \mathrm{~km}$ to 410 $\mathrm{km}$. And the value of $I_{o p}^{\prime} / I_{R}$ and $I_{o p}^{\prime} / I_{\text {res }}^{\prime}$ in terms of $\theta_{s}$ are shown in Figure 5 and Figure 6 correspondingly. The region above the threshold (dotted line) corresponds to the attack angle that leads to the misoperation of the relay. It is worth noting that when the differential protection is equipped with line charging current compensation and the compensation reduces the effect of line charging completely, the results in the previous section with minimal line charging are applicable.

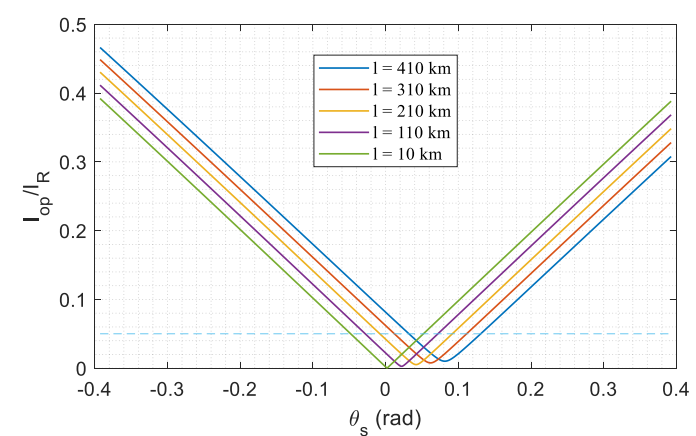

Figure 5: Percentage Line Differential, $I_{o p}^{\prime} / I_{R}$ in terms of the length of the line

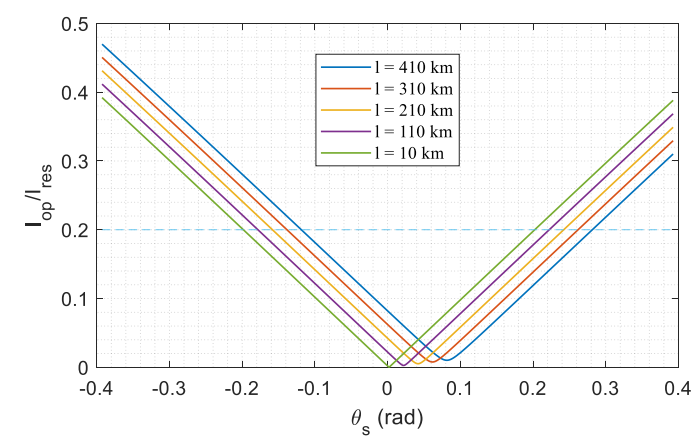

Figure 6: Percentage Line Differential, $I_{o p}^{\prime} / I_{\text {res }}^{\prime}$ in terms of the length of the line

\section{Alpha-plane Line differential Protection}

Another type of line differential protection is the alpha-plane line differential protection. This method is based on transmitting the measured current phasor from one end of the line to the other and taking the ratio of the current phasors at the two ends of the line. For an ideal line and neglecting the capacitive current of the line, this ratio will be exactly -1.0 . If there is an internal fault in the line the ratio of the current phasor will be different than -1.0. Because of the capacitive current of the line, the ratio of the currents will deviate from -1.0. Therefore, a region of restraint and this relay will trip if:

$$
\begin{gathered}
I_{r e s} / I_{R}>K_{3} \\
\text { AND } \alpha \notin \Omega_{4}
\end{gathered}
$$

In which,

$$
\begin{gathered}
\alpha=i_{2} / i_{1} \\
I_{\text {res }}=\frac{1}{2}\left(\left|i_{1}\right|+\left|i_{2}\right|\right)
\end{gathered}
$$

$I_{R}$ is the rated current of the transmission line.

$K_{3}$ is a user-defined constant, and $\Omega_{4}$ is a userdefined restraint region in the complex plane, which is defined by $R_{\text {in }}, R_{\text {out }}$ and $\theta_{\Omega 4}$. This is illustrated in Figure 7. 


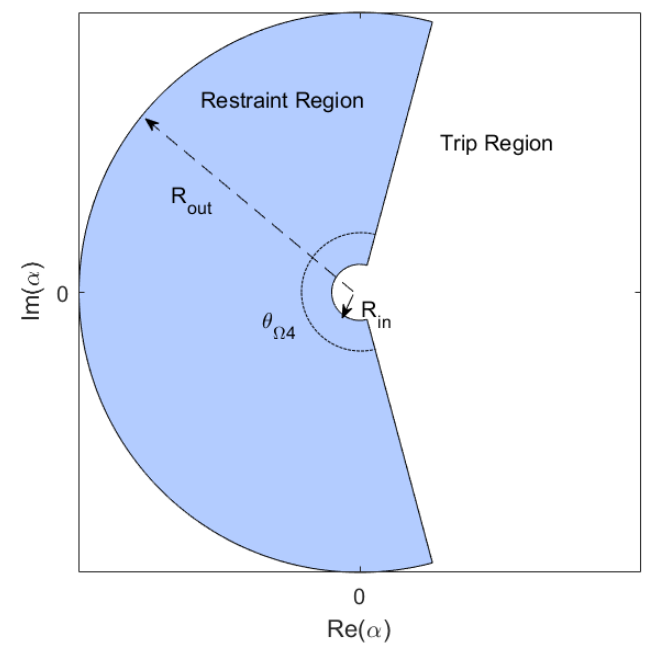

Figure 7: Alpha-plane Characteristics

\subsection{Line charging is minimal}

For short lines, $B \approx 0$, if there is no internal fault and the magnitude of the current is rated current. Then the restraining current can be calculated as:

$$
I_{r e s}^{\prime}=\frac{1}{2}\left(\left|i_{1}\right|+\left|i_{2} e^{j \theta_{s}}\right|\right)=\frac{1}{2}\left(\left|i_{1}\right|+\left|i_{2}\right|\right)=I_{r e s}=I_{R}
$$

Therefore the expression (4) will not be affected by the GPS spoofing attack. In other words, if there is no internal fault, the current flowing through the line is less than the rated value $I_{R}$ and $K_{3}>1$, the condition will not be satisfied regardless of the attack angle.

The $\alpha$ in expression (5) is calculated as:

$$
\alpha^{\prime}=\frac{i_{2}^{\prime}}{i_{1}}=\frac{y\left(v_{2}-v_{1}\right) e^{j \theta_{s}}}{y\left(v_{1}-v_{2}\right)}=-e^{j \theta_{s}} \notin \Omega_{4} \rightarrow\left|\theta_{s}\right|>\frac{\theta_{\Omega 4}}{2}
$$

Therefore, this condition will be satisfied if the spoofing attack angle is inside the trip region. In summary, when there is no fault inside the protected line, the misoperation of alpha-plane line differential protection due to GPS spoofing attack is unlikely, because the value of the expression (4) is not affected.

\subsection{Line charging is not minimal}

If $B$ is not minimal, the performance of the alphaplane method is studied numerically. The parameters of the line and relay are:

$$
\begin{gathered}
y(l)=1 /((0.025+0.2 i) l) \\
B(l)=0.001 l \\
v_{2}(l)=1.0 \\
v_{1}(l)=v_{2}(l) \exp (0.001 i * l) \\
I_{R}(l)=\left|y(l)\left(v_{1}(l)-v_{2}(l)\right)\right|
\end{gathered}
$$

$$
\begin{gathered}
\theta_{\Omega 4}=\frac{7}{6} \pi \\
R_{\text {in }}=0.3, R_{\text {out }}=3.0
\end{gathered}
$$

where $l$ is the length of the line.

In Figure 8, the value of $I_{r e s}^{\prime} / I_{R}$ in (4) for the alphaplane method is plotted. It is clear that the value of $I_{\text {res }}^{\prime} / I_{R}$ is constant when $\theta_{s}$ varies. Therefore, this condition will not be affected by the spoofing attack. When there is no internal fault in the line, the misoperation due to a GPS spoofing attack is unlikely. However, during a through fault this value may exceed the setting and the relay may misoperate during a GPS spoofing attack.

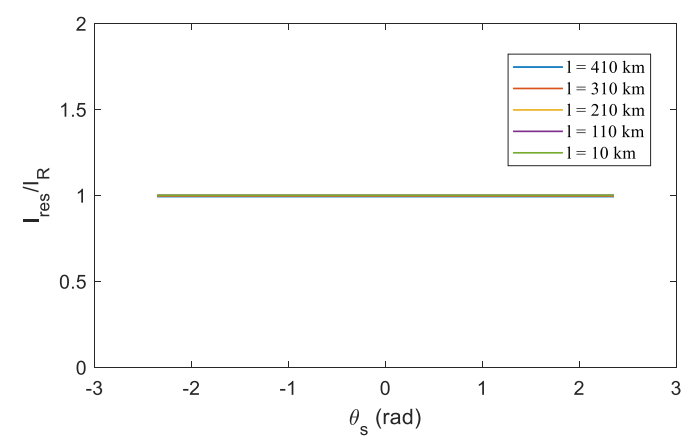

Figure 8 Value of $I_{\text {res }}^{\prime} / I_{R}$ in Alpha-plane

In Figure 9, the points corresponding to $\alpha^{\prime}$ under different angles are plotted. Also, the length of the line changes from $10 \mathrm{~km}$ to $410 \mathrm{~km}$. The magnitude of $\alpha^{\prime}$ remains constant and the angle of $\alpha^{\prime}$ varies as $\theta_{s}$ changes. For most points with $\left|\theta_{s}\right|<\theta_{\Omega 4}, \alpha^{\prime}$ is in the restraint region. However, line charging will shift the angles of the points, and push them clockwise. The zoomed-in view is shown in Figure 10. This lead to the unsymmetrical behavior for $\theta_{s}=7 / 12 \cdot \pi$ and $\theta_{s}=-7 / 12 \cdot \pi$. When $\theta_{s}=-7 / 12 \cdot \pi$ the point corresponds to $\alpha^{\prime}$ will enter the trip region. In addition, as the length of the line increase, which indicates the increase of the line charging, the point will move away from the boundary. In other words, for a longer transmission line, the effective attack angle leading to the relay misoperation is smaller. 


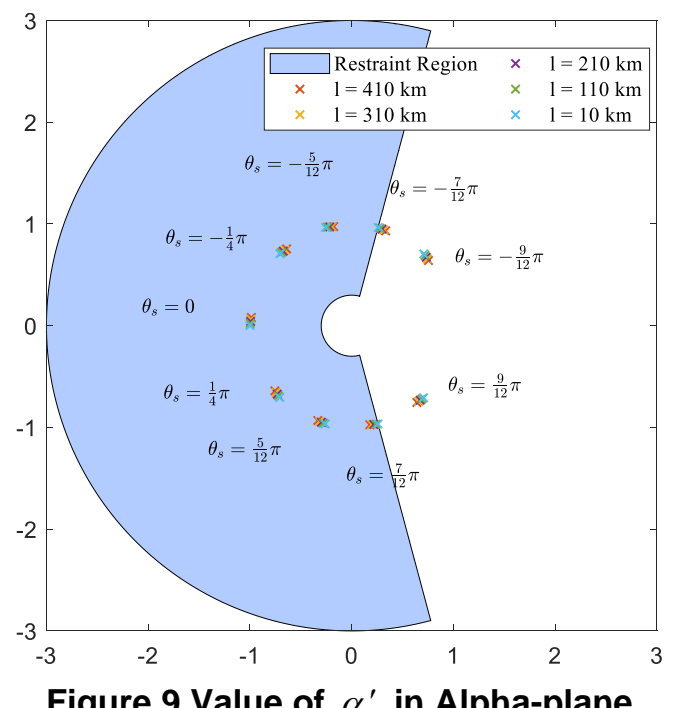

Figure 9 Value of $\alpha^{\prime}$ in Alpha-plane
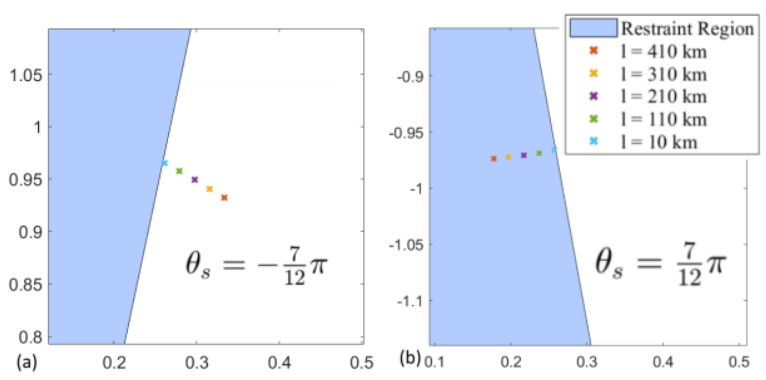

Figure 10 Value of $\alpha^{\prime}$ in Alpha-plane, near the trip region boundaries

\section{GPS Spoofing Attack Detection and Recovery}

To reduce the effect of GPS spoofing attack on line differential protection schemes, a quasi-dynamic stateestimation-based method is used to detect the attack. In addition, this method can recover the actual PMU measurement data from the compromised measurements.

\subsection{Quasi-Dynamic State Estimation}

The quasi-dynamic state estimation (QDSE) algorithm utilizes measurements to obtain an optimal estimate of the states of the grid. In this paper, we formulate the quasi-dynamic state estimator as an unconstrained state estimation problem[14], [15]:

$$
\begin{aligned}
& \min _{x(t)} \chi^{2}=\sum_{j=1}^{M}\left(\frac{h_{j}(x(t))-z_{j}(t)}{\sigma_{j}}\right)^{2} \\
& P=1-\operatorname{Pr}\left(\chi^{2}, v\right)
\end{aligned}
$$

where $x(t)$ is the state vector, $z_{j}(t)$ is the $j$ th measurement value, $\sigma_{j}$ is the standard deviation relating to the measurement, and $h_{j}(x(t))$ provides the measurement $j$ as a function of the state. Note that in the case of QDSE, the function $h_{j}(x(t))$ is based on the transient model of the power system. The dimension of $x(t)$ equals $N$, including, bus voltage magnitudes, phase angles, generator speed, etc. The dimension of $z(t)$ equals $M$, including, voltage phasor measurement, current phasor measurement, generator speed measurement, virtual measurement, etc. $\chi^{2}$ describes the consistency between the measurement value and the estimation model. $\operatorname{Pr}\left(\chi^{2}, v\right)$ is the Chi-square cumulative probability distribution function, and $P$ is the confidence level, $v$ is the difference between the number of measurements and states (degree of freedom). A low confidence level corresponds to inconsistency between measurement value and the measurement model. This can be utilized to detect the internal fault of the device and bad data in the measurements[14].

To utilize more measurements related to the electromechanical dynamics in the system, the quasidynamic state estimation (QDSE) method is proposed. The expression $h_{j}(x(t))$ uses the transient model of the system resulting in dynamic relationships between measurements and states. For example, the variation of mechanical speed and the angle of rotors in generators and motors are considered in QDSE. Therefore, the proposed method increases the number of measurements. In general, more measurements lead to increased robustness of the state estimation against noise and attacks. In addition, the quasi-dynamic state estimation uses a three-phase model. The incorporation of the three-phase model ensures accurate estimation results even though when accommodating an unbalanced and asymmetric system [17]. The inclusion of the transient model enables QDSE to yield more accurate results[18], compared with traditional state estimation. The well-known $\chi^{2}$ test is used to verify the performance of state estimation. The $\chi^{2}$ value is used to calculate the confidence level $P$. When the confidence level $P$ drops below a predefined threshold $P_{t h}$, then the inputs of the state estimation are abnormal. The root cause is determined by hypothesis testing. The phasor measurements in the suspected substation under GPS spoofing attack are replaced with magnitude measurements. The modified measurements and remaining measurements are transmitted to QDSE and the confidence level is computed again, and the value is $P^{\prime}$. A GPS spoofing attack is identified if this hypothesis test passes, i.e. $P^{\prime}>P_{t h}$. The estimated measurements 
are evaluated without the effect of spoofing attack and these recovered measurements are streamed to relays. Meanwhile, the attack angle can be revealed through another approach: for all phasor measurements coming from a group of PMUs that are connected to the same GPS clock, we introduce an unknown time shift expressed in a phase angle. Each phasor in this group becomes:

$$
\tilde{A}^{m}=\tilde{A}^{\text {true }} e^{j \theta}
$$

The value of the introduced phase angle represents the phase shift caused by the GPS spoofing. Note this approach introduces one unknown variable for each group of PMUs connected to the same GPS clock. In general, a GPS attack is local and in most cases there is one GPS clock for each substation. Thus the approach introduces an additional unknown, one for each substation. The state estimation provides the value of this unknown and therefore quantifies the attack.

\subsection{Numerical Results}

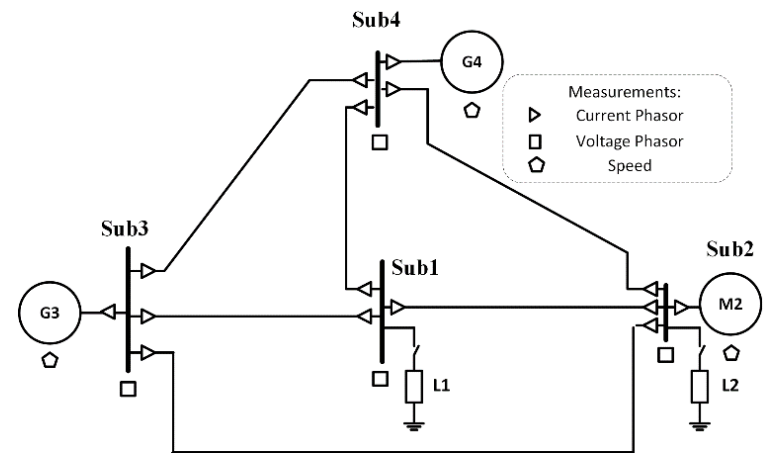

Figure 11: Illustrative Test Case

The quasi-dynamic state estimation method is utilized to detect and eliminate the impact of GPS spoofing attack on line differential protection. The test case used for illustration is shown in Figure 11. All the measurements from 4 substations are depicted in the figure, including voltage measurement, current measurement, and rotor speed measurements. The target of the attack is substation 1, therefore all measurements captured at substation 1 will be affected, including $I_{12}, I_{13}, I_{14}$ and $V_{1}$. The attack angle follows the equation (8), where $u(t)$ is the unit step function. In practice, attackers are likely to ramp up $\theta_{s}$ to escape abrupt change detection so a ramp function is also included. The investigated protection zone includes the transmission line from substation 1 to substation 2. A percentage differential protection scheme is implemented with measurement $I_{12}$ from substation 1 and $I_{21}$ from substation 2 .

$$
\theta_{s}=\frac{\pi}{12}(t-0.5)(u(t-0.5)-u(t-1.5))
$$

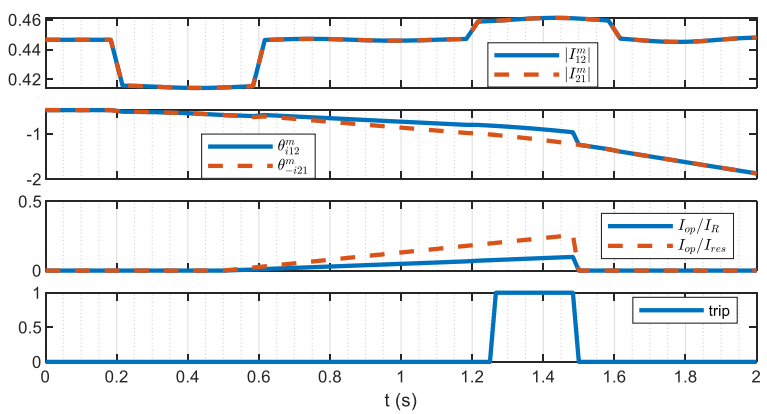

Figure 12: Relay Performance under Attack

The performance of the relay is shown in Figure 12. The magnitude and angle of measurement $I_{12}^{m}, I_{21}^{m}$ are plotted in the first two rows. It is obvious that the difference between $\theta_{i 12}^{m}$ and $\theta_{-i 21}^{m}$, which are the angle of $I_{12}^{m}$ and $\left(-I_{21}^{m}\right)$ correspondingly, increases after the attack starts. The magnitude of $I_{o p}$ increases as a result. Though there is no internal fault of the line, the relay issues a trip decision after $I_{o p} / I_{R}$ and $I_{o p} / I_{\text {res }}$ exceed the threshold. The misoperation of line differential protection due to GPS spoofing attack is verified.

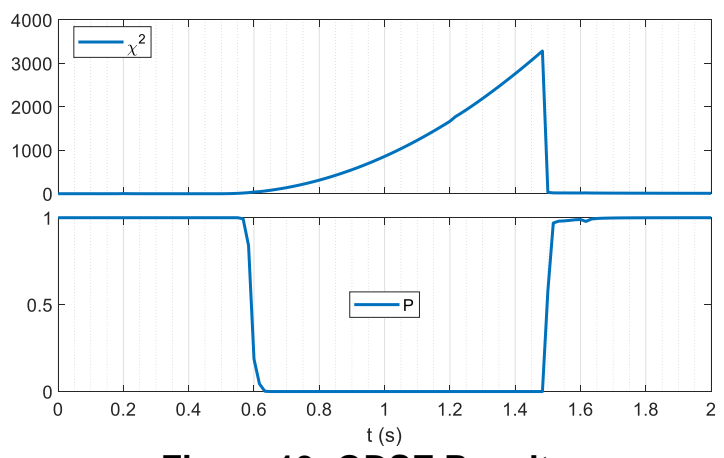

Figure 13: QDSE Results

To eliminate the impact of GPS spoofing attack on the relay, the QDSE method utilizes the measurements from all 4 substations and estimates the states of the network, including the bus voltages, generator rotor angles, etc. Then, estimated measurements are generated from the estimated states. $\hat{I}_{12}$, for example, is the estimated measurements, which can verify the field measurement $I_{12}^{m}$. The overall distance between measurements and field measurements are denoted as $\chi^{2}$, which is plotted in Figure 13. The increase of $\chi^{2}$ and decrease of $\mathrm{P}$ indicate the discrepancy between measurement value and the system model. Since the 
major discrepancy comes from measurements at substation 1, a hypothesis test is conducted with an alternative formulation of the QDSE. The complex measurements at substation are replaced with magnitude only:

$$
\begin{aligned}
& V_{1 r}^{m}, V_{1 i}^{m} \rightarrow\left(\left|V_{1}^{m}\right|\right)^{2}=\left(V_{1 r}^{m}\right)^{2}+\left(V_{1 i}^{m}\right)^{2} \\
& I_{12 r}^{m}, I_{12 i}^{m} \rightarrow\left(\left|I_{12}^{m}\right|\right)^{2}=\left(I_{12 r}^{m}\right)^{2}+\left(I_{12 i}^{m}\right)^{2} \\
& I_{13 r}^{m}, I_{13 i}^{m} \rightarrow\left(\left|I_{13}^{m}\right|\right)^{2}=\left(I_{13 r}^{m}\right)^{2}+\left(I_{13 i}^{m}\right)^{2} \\
& I_{14 r}^{m}, I_{14 i}^{m} \rightarrow\left(\left|I_{14}^{m}\right|\right)^{2}=\left(I_{14 r}^{m}\right)^{2}+\left(I_{14 i}^{m}\right)^{2}
\end{aligned}
$$

The result of the hypothesis test is shown in Figure 14. The $\chi^{2}$ value remains low and the confidence level is close to 1 . Therefore, the cause of the abnormality in state estimation is verified and the spoofing attack is detected. In addition, the estimated measurements of the QDSE are used to recover the affected measurements. They are provided for the relay and the performance is shown in Figure 15. The value of $I_{o p} / I_{R}$ and $I_{o p} / I_{\text {res }}$ remain minimal and misoperation is avoided.

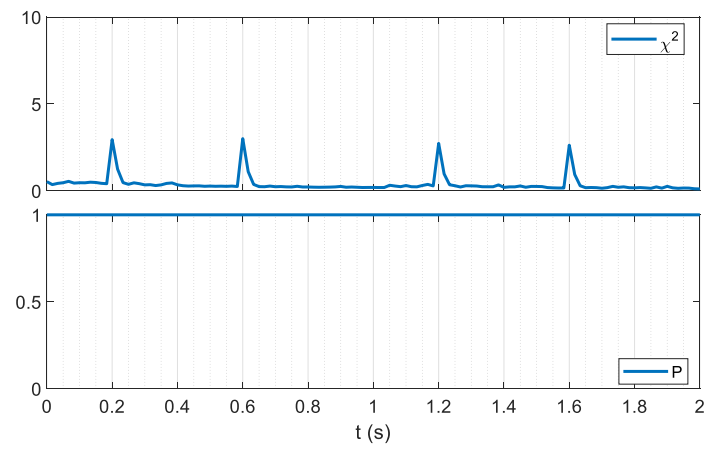

Figure 14: QDSE Result with Alternative Formulation

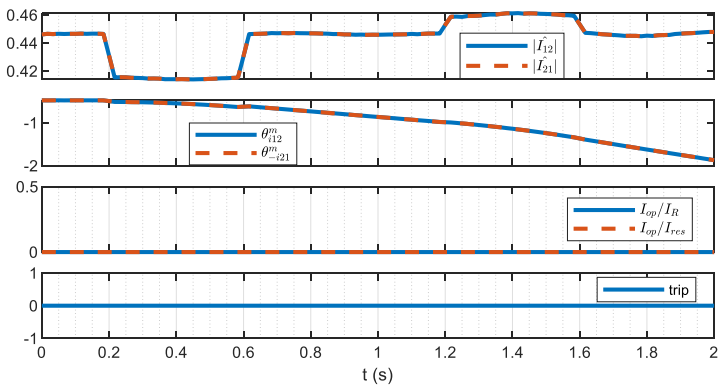

Figure 15: Relay Performance with Recovered Measurements

In addition, we can enhance the state estimation by introducing an additional state to represent the phase shift caused by the GPS spoofing. Therefore, all the measurements model corresponding to voltage/current measurements in substation 1 are revised as:

$$
h_{i}^{\prime}(x(t))=h_{i}(x(t)) \exp \left(j \theta_{s}\right)
$$

The solution of the revised QDSE has one addition estimation state $\hat{\theta}_{s}$, which is shown in Figure 16. The estimated phase shift is highly consistent with the actual attack angle. Therefore, the GPS spoofing attack is quantified.
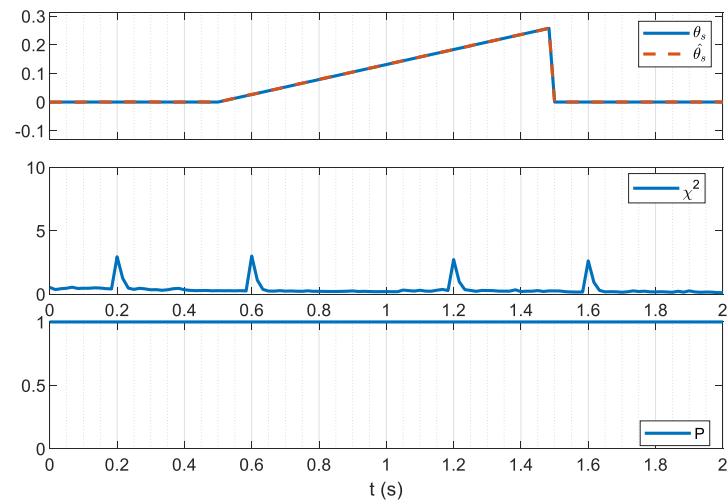

Figure 16: QDSE Result with Attack Angle as State

\section{Conclusion}

This paper presents the effect of GPS spoofing attack on PMU based transmission line differential protective relays. The misoperation of the relay is observed in percentage line differential protection. If the spoofing attack angle is larger than the threshold, which is determined by the relay characteristics, the relay will trip even though there is no internal fault in the line. Therefore, the malicious attacker can substantially disturb the normal operation of the power grid. These results from numerical simulations can be further enhanced with real transmission line parameters and system states, which allow protection engineers to assess the vulnerability of a particular system to possible attacks. The inclusion of negative-sequence and zerosequence components will be studied in future work. In addition, this paper presents a quasi-dynamic stateestimation-based method to detect GPS spoofing attacks and recover the actual measurements. This method utilizes a dynamic model of the system and higher redundancy measurements such as three-phase measurements, generator speed, virtual measurements, etc. The numerical results show that the method can detect the attack immediately. This method also enables the recovery of corrected measurements, eliminating the misoperation of the line differential protection scheme. 


\section{References}

[1] T. Yasmin Mina, S. Bhamidipati, and G. Xingxin Gao, "GPS spoofing detection for the power grid network using a multireceiver hierarchical framework architecture," Navigation, vol. 66, no. 4, pp. 857-875, Dec. 2019.

[2] K. Borre, D. M. Akos, N. Bertelsen, P. Rinder, and S. H. Jensen, A software-defined GPS and Galileo receiver: a single-frequency approach. Springer Science \& Business Media, 2007.

[3] T. E. Humphreys, B. M. Ledvina, M. L. Psiaki, B. W. O'Hanlon, and P. M. Kintner, "Assessing the spoofing threat: Development of a portable GPS civilian spoofer," 2008.

[4] N. Eastland and V. M. Venkatasubramanian, "Impact of GPS Spoofing on HVDC Modulation," in Proceedings of the 53rd Hawaii International Conference on System Sciences, Jan. 2020, pp. 31963203.

[5] X. Jiang, J. Zhang, B. J. Harding, J. J. Makela, and A. D. Dominguez-Garcia, "Spoofing GPS Receiver Clock Offset of Phasor Measurement Units," IEEE Trans. Power Syst., vol. 28, pp. 3253-3262, Aug. 2013.

[6] J. De La Ree, V. Centeno, J. S. Thorp, and A. G. Phadke, "Synchronized Phasor Measurement Applications in Power Systems," IEEE Trans. Smart Grid, vol. 1, no. 1, pp. 20-27, Jun. 2010.

[7] F. Aminifar, M. Fotuhi-Firuzabad, A. Safdarian, A. Davoudi, and M. Shahidehpour, "Synchrophasor Measurement Technology in Power Systems: Panorama and State-of-the-Art," IEEE Access, vol. 2, pp. 1607-1628, 2014.

[8] Y. Cui, F. Bai, Y. Liu, P. L. Fuhr, and M. E. MoralesRodriguez, "Spatio-Temporal Characterization of Synchrophasor Data Against Spoofing Attacks in Smart Grids," IEEE Trans. Smart Grid, vol. 10, no. 5, pp. 5807-5818, Sep. 2019.

[9] C. Konstantinou, M. Sazos, A. S. Musleh, A. Keliris, A. Al-Durra, and M. Maniatakos, "GPS spoofing effect on phase angle monitoring and control in a real-time digital simulator-based hardware-in-the-loop environment," IET Cyber-Phys. Syst. Theory Appl., vol. 2, no. 4, pp. 180-187, Dec. 2017.

[10] Joe-Air Jiang, Jun-Zhe Yang, Ying-Hong Lin, ChihWen Liu, and Jih-Chen Ma, "An adaptive PMU based fault detection/location technique for transmission lines. I. Theory and algorithms," IEEE Trans. Power Deliv., vol. 15, no. 2, pp. 486-493, Apr. 2000.

[11] H. Miller, J. Burger, N. Fischer, and B. Kasztenny, "Modern line current differential protection solutions," in 2010 63rd Annual Conference for Protective Relay Engineers, College Station, TX, USA, Mar. 2010, pp. 1-25, Accessed: Sep. 19, 2020. [Online]. Available: http://ieeexplore.ieee.org/document/5469504/.

[12] D. M. E. Ingram, P. Schaub, D. A. Campbell, and R. R. Taylor, "Evaluation of Precision Time synchronisation methods for substation applications," in 2012 IEEE International Symposium on Precision Clock Synchronization for Measurement, Control and Communication Proceedings, San Francisco, CA, USA, Sep. 2012, pp. 1-6, Accessed: Sep. 20, 2020. [Online]. http://ieeexplore.ieee.org/document/6336630/.

[13] A. K. Mattei, W. M. Grady, P. J. Caspary, and S. A. McBride, "Detection of time spoofing attacks on GPS synchronized phasor measurement units," in 2016 69th Annual Conference for Protective Relay Engineers (CPRE), College Station, TX, USA, Apr. 2016, pp. 18, Accessed: Sep. 20, 2020. [Online]. Available: http://ieeexplore.ieee.org/document/7914884/.

[14] A. P. S. Meliopoulos et al., "Dynamic State EstimationBased Protection: Status and Promise," IEEE Trans. Power Deliv., vol. 32, no. 1, pp. 320-330, Feb. 2017.

[15] J. Xie and A. P. S. Meliopoulos, "Sensitive Detection of GPS Spoofing Attack in Phasor Measurement Units via Quasi-Dynamic State Estimation," Computer, vol. 53, no. 5, pp. 63-72, 2020.

[16] H. Wen, P. Y.-R. Huang, J. Dyer, A. Archinal, and J. Fagan, "Countermeasures for GPS signal spoofing," in ION GNSS, 2005, vol. 5, pp. 13-16.

[17] B. Xie, A. P. S. Meliopoulos, C. Zhong, Y. Liu, L. Sun, and J. Xie, "Distributed Quasi-Dynamic State Estimation Incorporating Distributed Energy Resources," in 2018 North American Power Symposium (NAPS), Fargo, ND, Sep. 2018, pp. 1-6, Accessed: Jan. 29, 2020. [Online]. Available: https://ieeexplore.ieee.org/document/8600662/.

[18] B. Xie et al., "A Performance Comparison Study of Quasi-Dynamic State Estimation and Static State Estimation," in 2020 IEEE Power \& Energy Society General Meeting (PESGM), Montreal, 2020, pp. 1-5. 